\title{
Indexing k-mers in Linear-space for Quality Value Compression
}

\author{
Yoshihiro Shibuya ${ }^{1,2}$ and Matteo Comin ${ }^{1}$ \\ ${ }^{1}$ Department of Information Engineering, University of Padua, via Gradenigo 6B, Padua, Italy \\ ${ }^{2}$ Laboratoire d'Informatique Gaspard-Monge (LIGM), University Paris-Est Marne-la-Vallée, \\ Bâtiment Copernic - 5, bd Descartes, Champs sur Marne, France
}

Keywords: $\quad$ k-mers, Indexing, Quality Score, Read Compression.

\begin{abstract}
Many bioinformatics tools heavily rely on k-mer dictionaries to describe the composition of sequences and allow for faster reference-free algorithms or look-ups. Unfortunately, naive k-mer dictionaries are very memory inefficient, requiring very large amount of storage space to save each k-mer. This problem is generally worsened by the necessity of an index for fast queries. In this work we discuss how to build an indexed linear reference containing a set of input k-mers, and its application to the compression of quality score in FASTQ files. Most of the entropy of sequencing data lies in the quality scores, and thus they are difficult to compress. Here, we present an application to improve the compressibility of quality values while preserving the information for SNPs calling. We show how a dictionary of significant k-mers, obtained from SNPs databases and multiple genomes, can be indexed in linear space and used to improve the compression of quality value. Availability: the software is freely available at https://github.com/yhhshb/yalff.
\end{abstract}

\section{INTRODUCTION}

The compression of DNA is usually as simple as assigning a two bit encoding to each of the four bases. This encoding achieve almost similar results to standard lossless compressors (Malysa et al., 2015). On the other hand, the quality values, produced by sequencing technologies, span a wider range of values, and when compressed they can sum up to about $70 \%$ of the total space to encode a FASTQ file (Greenfield et al., 2016). Quality values are usually encoded using the Phred system (Ewing et al., 1998). Quality values are often essential for assessing sequence quality, mapping reads to a reference genome, detecting mutations for genotyping, assembling genomic sequences, reads clustering (Comin et al., 2014; Comin et al., 2015) and comparison (Schimd and Comin, 2016).

Quality scores are more difficult to compress due to a larger alphabet (63-94 in original form) and intrinsically have a higher entropy (Yu et al., 2015). With lossless compression algorithms and entropy encoders reaching their theoretical limits and delivering only moderate compression ratios (Bonfield and Mahoney, 2013), there is a growing interest to develop lossy compression schemes to improve compressibility further.

To further reduce the file sizes, Illumina proposed a binning method to reduce the number of differ- ent quality values from 42 to 8 (Illumina8bin, 2011). With this proposal, Illumina opened the doors for allowing lossy compression of the quality values. Another approach called P-Block (Cánovas et al., 2014) involves local quantization so that a representative quality score replaces a contiguous set of quality scores that are within a fixed distance of the representative score. Similarly, the R-Block (Cánovas et al., 2014) scheme replaces contiguous quality scores that are within a fixed relative distance of a representative score. Other lossy approaches improve compressibility and preserve higher fidelity by minimizing a distortion metric such as mean-squared-error or L1-based errors (Qualcomp and QVZ) ((Malysa et al., 2015) (Ochoa et al., 2013)). The drawback of lossy compression of quality values is that downstream analysis could be affected by the loss incurred with this type of compression. This could be the case for the above methods that process only the string of quality scores, without considering the DNA sequence associated to the read. However, (Yu et al., 2015), (Ochoa et al., 2017) and (Greenfield et al., 2016) showed that quality values compressed with more advanced methods could achieve not only a better performance in downstream analyses than Illumina-binned quality values, but even better performance than the original quality values in some cases because these methods remove noise from the data. 
The most promising methods are those using both sequence and quality information. Leon (Benoit et al., 2015) constructs a reference from the input reads in the form of a bloom filter compressed de-Bruijn graph and then maps each nucleotide sequence as a path in the graph. If a base is covered by a sufficiently large number of k-mers from the reference its quality is set at a fixed high value. Among the most interesting tools, Quartz (Yu et al., 2015), similarly to Leon, relies on an external reference to decide if a given nucleotide is wrong or not. This reference database is implemented as list of $2497777428 \mathrm{k}$-mers stored explicitly, that requires 24GB when gzipped. Similarly, GeneCodeq (Greenfield et al., 2016) also has a list of k-mers as ground truth, but the algorithm involved during quality compression, i.e. smoothing, is more complex than Quartz. Each base has its associated error probability recalculated using a Bayesian framework and the smoothing takes place only if the new quality is greater than the old one. Both Quartz (Yu et al., 2015) and GeneCodeq (Greenfield et al., 2016) require a machine with at least $32 \mathrm{~GB}$ of RAM, because of the size of the reference database. In (Shibuya and Comin, 2018) we have proposed a method for quality value compression based on a single reference genome. However, the authors of (Yu et al., 2015) demonstrated that the use of significant k-mers coming from multiple genomes or SNPs databases, are beneficial for quality score compression and SNPs calling. Hence, in this paper we explore the use of multiple sources of mutations, i.e. SNP databases, for quality value compression.

The most common procedure to obtain a reference list of k-mers from a set of sequences is by a k-mer counting procedure. Most of the existing implementations rely on some hashing scheme. The hashing usually involves a simple application of a hash function to each k-mer to obtain a numeric value which is then used to access a hash table where the actual counters are kept. There exist efficient implementations of hashing functions which focus on optimizing and accelerating the generation of the hash value by using the previous computed hash like in (Mohamadi et al., 2016) or (Girotto et al., 2018b; Girotto et al., 2018a). On the other hand, a very fast implementation of a hash table can be found in Jellyfish (Marçais and Kingsford, 2011) which uses a lock-free table allowing access from multiple threads if they do not collide in the same bucket for writing operations. Another approach is found in (Rizk et al., 2013) and involves the use of minimizers, a type of Locality Sensitive Hashing to efficiently group similar k-mers into buckets.

The next step usually involves some sort of sorting and indexing for allowing fast retrieval of some particular k-mer (or its neighbors) and link this information to the position in the original sequence. This step is necessary whenever the counting procedure is the first step of a more complex pipeline. It is not so uncommon for some applications to index a sequence using the positions of each of its k-mers (Greenfield et al., 2016; Shajii et al., 2016). While this approach is advantageous when a proper counting of the k-mers is required it is not necessary in general when the final goal is to support set queries and/or retrieval of a specific sequence or its position inside the original string. For this purpose, it is generally possible to strip a dictionary of k-mers of the counters to obtain a smaller representation. Unfortunately, even in the hypothesis of removing all the under-represented k-mers and all counters, the space required by a full table is still high. For example, LAVA (Shajii et al., 2016) uses a databases of k-mers extracted from dbSNP (Sherry et al., 2001), because of the large size of this database it requires 60GB of ram. In fact, for a DNA sequence of length $n$ the potential number of k-mers is $n-k+1$ each of length $k$ for a total amount of space in the order of $O(k(n-k+1))=O(k n)$ bytes.

If the k-mer table has to be transmitted a naive solution would be to compress it using a standard compressor such as gzip or $\mathrm{xz}$. This procedure only works for moving the database from one place to another because to fully restore its functionality it must be decompressed anyway. Another question is how efficient the standard compressors (or an two bit encoding) are in reducing the size of the table. What is the best way to compress a set of k-mers? Is it possible to use them for an optimal compression scheme removing redundancy and exploiting the peculiar structure of each k-mer and its relation with the others?

To answer this question in this work we analyze a particular application of $\mathrm{k}$-mer dictionaries, that is the compression of quality scores. In this work we investigate how to construct a linear string, or set of strings, that contains all input k-mers. In particular we will consider a special set of k-mers extracted from popular SNPs databases like dbSNP (Sherry et al., 2001) or Affymetrix Genome-Wide Human SNP Array 6.0. In the following sections we present an application of a reassembly procedure to reduce a dictionary of kmers into a set of sequences guaranteed to contain all the k-mers. This procedure will allow us to store the whole dictionary in linear form reducing the memory requirements from $O(k n)$ to $O(n)$ without losing information. For querying the resulting dictionary we will index it with the FM-Index, and use the FM-index for quality value compression. 


\section{METHODS}

The main insights in order to reduce the size of the dictionary is that most of the information carried by a $\mathrm{k}$-mer stored explicitly is redundant. This intuition is easily explained by recalling the k-mer counting procedure itself. All the k-mers counted comes from a set sequences and the counting procedure is only necessary to remove the wrong ones. There is no need to keep the k-mers explicitly stored to answer simple yes/no queries over their set. Given two consecutive $\mathrm{k}$-mers it is possible to reassemble them into a single $(\mathrm{k}+1)$-mer thus reducing the storage requirement by k-1 bases.

\subsection{Dictionary Reassembly}

There exists two methods to build a dictionary string comprising all the k-mers needed for quality smoothing. The first is a k-mer counting procedure followed by an assembly (Figure 1 top left). If the counting is performed on a set of real datasets of reads then a filtering of the k-mers whose counter is below a certain threshold might be required to prevent false positives to be used as ground truth, as in (Yu et al., 2015). The filter is effective only if the datasets used have a very high coverage, leading to increased memory and time requirements for this step.

The reassembly step can be carried out on almost all dictionaries regardless of the tools used to generate them, leading to a linear sequence, or set of sequences, that contains all the input k-mers. This requirement is what makes this step different from a common de-novo assembly where the k-mers that do not align well with the graph are discarded leading to k-mers not present in the final reconstructed sequence. In general, this procedure will output more than one sequence because of the uniqueness of each $\mathrm{k}$-mer in the resulting strings. Some redundant k-mers could allow for the concatenation of multiple contigs into one. The strings obtained are generally stored into a simple FASTA file where the identifiers can lead to unwanted memory usage especially if there are a very large number of short contigs. The memory requirements can worsening during the indexing of the FASTA file by adding information to account for the boundaries of each sequence. Simply concatenating each conting to each other produce k-1 spurious k-mers for each junction which might be a source of potential false-positives during the application of quality value compression. The software used to perform this step is the greedy ProphAsm assembler (ProphAsm) used in the Prophyle metagenomic suite (Břinda, 2016; Břinda et al., 2017). In contrast to the similar software BCalm (Chikhi et al., 2016) it doesn't stop at each discordant new k-mer producing less and longer contigs.

If the previous method does not lead to a significant reduction of space compared to an already available reference genome (Figure 1 bottom left) then the latter one can be the right choice. The previous method of construction will probably lead to a set of strings very similar to the reference genome of the organism even when applied in an optimal setting (when the k-mers can be aligned into few contigs). It is true that a counting procedure involving many sequences will include many variants in the final dictionary string but nonetheless it is very convenient to use a reference genome directly.

Both method can be extended for accounting for known lists of SNPs. The first one can include additional k-mers during the counting procedure and reassembly the whole dictionary back into a string. These additional k-mers can be extracted from a list of known SNPs, like in LAVA (Shajii et al., 2016), where for each SNP we can we take the k-mers that overlap with the SNP with the reference allele replaced by the alternate. If the starting point is an already assembled set of dictionary strings it is possible to simply add the relevant k-mers at the end of one contig by concatenation or by maintaining the list of known SNPs as is and load it together with the reference string. This work uses the first method because as described in the Results section the realigned dictionary contains significant k-mers coming from multiple human genomes, extracted from dbSNP (Sherry et al., 2001) and Affymetrix Genome-Wide Human SNP Array 6.0.

The problem of indexing a set of reference sequences in minute space, while providing full search capability, has been widely studied and efficient data structure are now available. The data structure chosen for this purpose is the FM-Index (Ferragina and Manzini, 2000; Ferragina and Manzini, 2005) which is based on the Burrows-Wheeler transform (BWT) (Burrows and Wheeler, 1994) of a sequence. The FM-index, and its variants, are now at the basis of many algorithms in the field of sequence analysis. For example, one of the most used tool for reads mapping, BWA ( $\mathrm{Li}$ and Durbin, 2010), is based on the FM-index and it requires as input the FM-index of the reference genome. For its simplicity, its relative easy installation and its widespread usage, we decided to use the BWA for indexing and querying our list of sequences. The FM-index will be used to search for $\mathrm{k}$-mers. The procedure to retrieve the position of a $\mathrm{k}$-mer is the enhanced backward search algorithm described in (Li and Durbin, 2009), that is also able to 


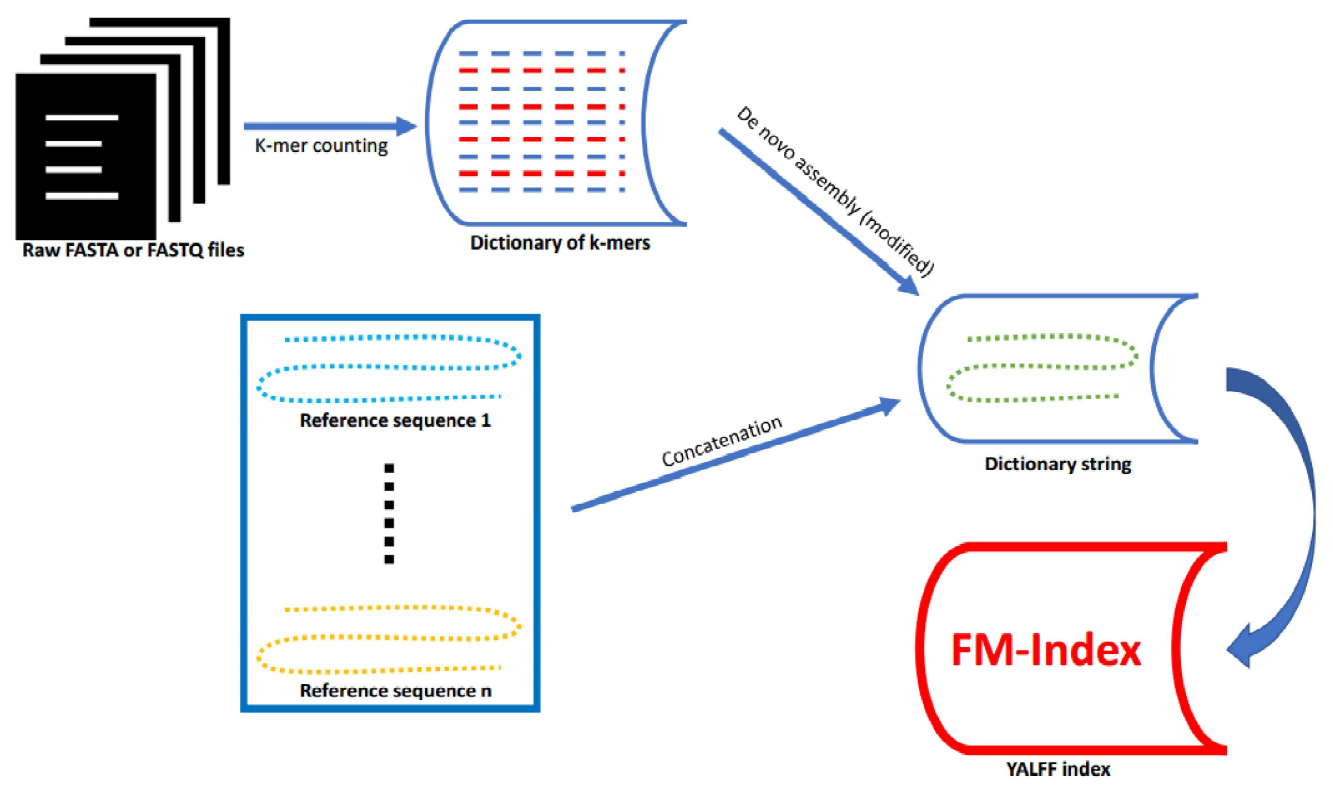

Figure 1: Examples of dictionary string construction. The first method employs a k-mer counting procedure, the second one uses an already available reference genome and other reference sequences. Additional information, e.g. regarding SNPs data, can be included in the dictionary string by concatenation. In the last step the dictionary string is indexed using an FM-Index.

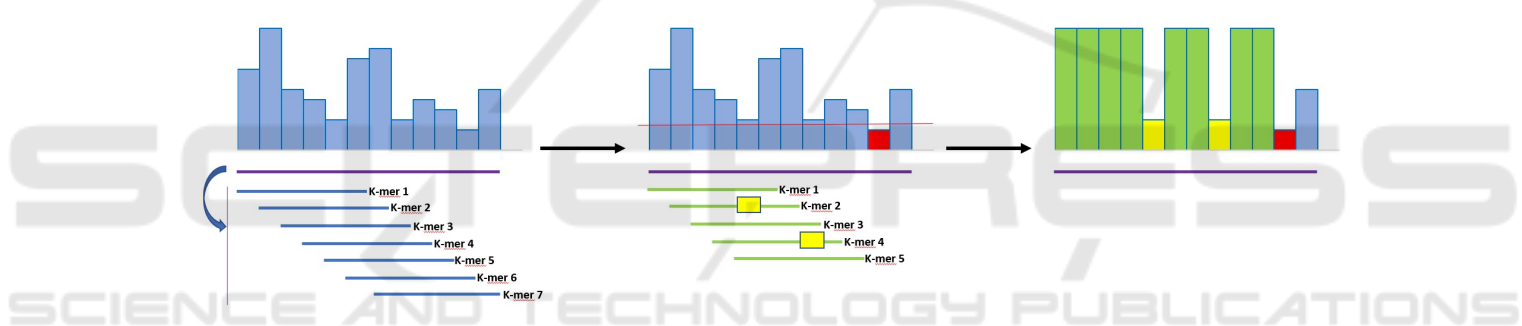

Figure 2: An example of quality smoothing by YALFF including both mismatches with the k-mers DB and low quality values.

account for mismatches. In our case we will search if a k-mer is present in the reference genome with up to one mismatch.

In our work for compressing quality scores (Shibuya and Comin, 2018) the use of BWA also had the collateral advantage of not requiring a separate indexed FASTA for compression instead sharing the same indexed reference genome for reads alignments. In this work the indexed sequences are not equivalent to a reference genome, but instead they represent a set of k-mers that uniquely identifies known variants from dbSNP and Affymetrix SNPs databases.

\subsection{Quality Value Compression based on k-mers}

We evaluate the quality of the k-mers dictionary for the compression of quality values. This work extends our previous work on YALFF (Shibuya and Comin, 2018) where we presented a novel algorithm for compressing quality strings without losing precision dur- ing SNPs calling. The main differences are in the reference sequence used for compression. In (Shibuya and Comin, 2018) we compress a FASTQ file using always a single reference genome (i.g. HG38), while in this study we build a sequence dictionary based on a set of significant k-mers that appear in multiple genomes coming from the 1000 Genome Project, the dbSNP, and the Affymetrix SNPs databases. To keep the paper self-contained here we briefly describe the YALFF algorithm.

The method YALFF employs a dictionary of kmers to assess if a base of a read is correct. A reads is decomposed into its constituent k-mers and they are searched in the reference dictionary. Given a base of the read, if all k-mers that cover the base are found in the dictionary then we assume that the base is correct and thus we can modify (smooth) the corresponding quality score to a fixed high value, see Figure 2 for an example. In the first version of YALFF, the database against which the k-mers are checked is the well known human reference genome hg38 in 
the form of a BWA index. A summary of the algorithm is illustrated in Figure 3. The threshold should be chosen depending on if it is necessary to avoid as much distortion as possible or if compression is considered much more important. As a rule of thumb a higher threshold maintains more quality values unchanged but this leads to an increase in the entropy of the output file. A good value found for this study was a quality value equal to the character' (apex) which corresponds to a probability of error of 0.25119 .

Quartz (Yu et al., 2015) is another FASTQ compressor which explicitly stores all the k-mers in a hash table. Quartz requires 25GB of RAM whereas YALFF only need 5.7GB. The k-mer counting procedure used to build the default database of Quartz was performed on multiple NGS reads coming from different individuals of the 1000 Genome Project and therefore contains more significant k-mers compared to the simple reference genome. Similarly in LAVA (Shajii et al., 2016), the authors use two popular SNPs databases like dbSNP (Sherry et al., 2001) and Affymetrix Genome-Wide Human SNP Array to select a set of k-mers that uniquely identify these mutations. Here, we will use these set of k-mers instead of a single reference genome.

All subsequent results use k equal to 32, the length used by other studies (Yu et al., 2015; Greenfield et al., 2016; Shibuya and Comin, 2018). 32 has been chosen because the k-mers should be long enough to ensure that the number of all possible k-mers is much larger than the number of unique $\mathrm{k}$-mers in the genome, so as to ensure incidental collisions between unrelated k-mers are rare. Also, k-mer length should ideally be a multiple of four, since a $4 \mathrm{bp}$ length DNA sequence can be represented by a single byte and discriminating between forward and reverse strand is not important in this application (it is only required a yes/no response to a query). A 32-mer satisfies these constraints (Yu et al., 2015; Greenfield et al., 2016); it is represented by a single 64-bit integer, with a relatively low probability of containing more than one sequencing error with Illumina sequences, as well as resulting in few k-mer collisions.

\subsection{Datasets, Pipeline and Parameters}

The dataset used in this study is a set of real reads (NA12878) from the 1000 Genomes Project (Consortium, 2012). Only the two paired end archives were used (namely SRR622461_1.filt.fastq.gz and SRR622561_2filt.fast.gz) for the evaluation, while the third containing unpaired reads were discarded. For validation, we used an up-to-date high-quality genotype annotation generated by the Genome in a
Bottle Consortium (Zook et al., 2014). The GIAB gold standard contains validated genotype information for NA12878, from 14 sequencing datasets with five sequencing technologies, seven read mappers and three different variant callers. To measure accuracy, we use loci in the SNP list which are also genotyped in the GIAB gold standard (so called high confident regions) (SNPs, 2018). This dataset has been widely used for benchmarking in other papers (Yu et al., 2015; Shajii et al., 2016),

One of the dictionary string used as a reference is the human genome reference FASTA file hg38. fa downloaded from (HG38, 2018). The other k-mers databases are generated from dbSNPs (Sherry et al., 2001) and Affymetrix Genome-Wide Human SNP Array 6.0. Although YALFF can be run on a normal laptop the ProphAsm assembler requires a powerful computer to load all the k-mers it needs to reassembly. For this reason all tests were performed on a 14 lame blade cluster DELL PowerEdge M600 where each lame is equipped with two Intel Xeon E5450 at $3.00 \mathrm{GHz}, 16 \mathrm{~GB}$ RAM and two 72GB hard disk in RAID-1 (mirroring).

The basic idea is to compare the compression results for the given dataset using both the original reference genome and the re-assembled dictionary of k-mers of dbSNPs and Affymetrix Genome-Wide Human SNP Array with YALFF's algorithm. The performance evaluation of the smoothing procedure alone compares the number of retrieved SNPs from a smoothed FASTQ to the ground truth associated to the original dataset, with each set of variants (stored in the output VCF file) compared against the consensus set of variants. Each evaluation comprises three metrics: Precision, Recall and F-Measure. Once these parameters have been computed for using both dictionaries they can be compared each other to assess how the additional variants inside the k-mers database can influence a smoothing algorithm and the linear representation of the k-mers set. The genotyping pipeline is implemented as a single bash script which uses bwa mem for alignments, bcftools for SNP calling and rtgplot for evaluation.

The exact commands are reported for each program used outside the evaluation pipeline:

- ./print_quartz_dict dec200.bin.sorted $\mid$ prophasm k 32 -i - -o <Output FASTA >

- cat $<$ Input file $>\mid$./YALFF -d hg38.fa -g \’ -t $1>$ $<$ Output file $>$

Where print_quartz_dict is a simple utility written in C that prints each k-mer of Quartz's binary dictionary to the standard output. 


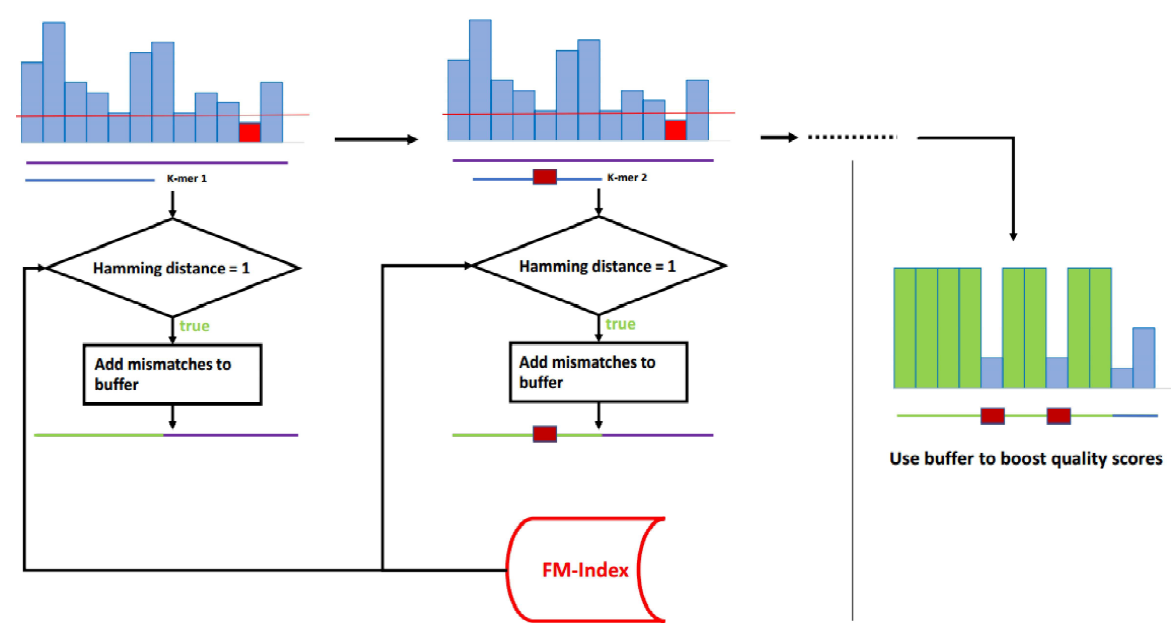

Figure 3: Diagram of YALFF's inner workings.

\section{RESULTS}

In this section the effects of the different k-mers databases are compared in terms of genotyping accuracy and compression of the database itself and the input FASTQ files. In order to give some context to the measurements related to the unprocessed input and Quartz are also reported from the YALFF paper.

\subsection{Effects on Genotyping Accuracy}

The performance evaluation of the algorithms compares the number of retrieved SNPs from a smoothed file to the ground truth. The latter comes with the original dataset and contains a set of variants validated using the genetic information of the parents whose child is the individual providing the source of the dataset. Each set of variants is stored in a VCF file analyzable with the software described before.

The benchmarking tools output the following values:

- True Positives (T.P.): All those variants that are both in the consensus set and in the set of called variants.

- False Positives (F.P.): All those variants that are in the called set of variants but not in the consensus set.

- False Negatives (F.N.): All those variants that are in the consensus set but not in the set of called variants.

These values are used to compute the following three metrics:

- Recall: This is the proportion of called variants that are included in the consensus set; that is, $R=$ T.P./(T.P. +F.N.),
- Precision: This is the proportion of consensus variants that are called by the variant calling pipeline; that is, $P=$ T.P./(T.P. +F.P.).

- F-Measure: The harmonic mean of precision and recall; that is, $F-$ Measure $=2 *(P * R) /(P+R)$

The following abbreviations are used in each table:

- None: The original FASTQ files without any further modification.

- Quartz: The result of smoothing the original input dataset with Quartz and its standard k-mers dictionary.

- YALFF_hg38: The result of smoothing the original input dataset with YALFF and the hg38 reference genome as a database of $\mathrm{k}$-mers.

- YALFF_Affy: YALFF when using the reassembled database of significant k-mers from Affymetrix Genome-Wide Human SNP Array 6.0 .

- YALFF_dbSNPs: YALFF when using the reassembled database of significant k-mers from dbSNPs.

- YALFF_All: YALFF when using the reassembled database of k-mers from hg38, dbSNPs and Affymetrix Genome-Wide Human SNP Array 6.0 .

In Table 1 are reported the results on the genotyping accuracy for the various tools.

Table 1 illustrates that Quartz is the most aggressive in terms of raw numbers. Compared to the original dataset it tends to add a very large number of True Positives and False Positives while greatly decreasing the False Negatives. This overall behavior would 
Table 1: Comparison of the number of True Positives, False Positives and False Negatives found by the SNP calling pipeline after smoothing the input reads with the various methods.

\begin{tabular}{|c|c|c|c|}
\hline Method & T.P. & F.P. & F.N. \\
\hline None & 2588159 & 219803 & 1493731 \\
Quartz & 2661218 & 237820 & 1420672 \\
YALFF_hg38 & 2603620 & 221368 & 1478264 \\
YALFF_Affy & 2610302 & 222017 & 1471583 \\
YALFF_dbSNP & 2645734 & 222820 & 1436155 \\
YALFF_All & 2652457 & 224616 & 1429433 \\
\hline
\end{tabular}

be more than adequate if well calibrated, but unfortunately it tends to degrade the Precision. On the other hand, the algorithm of YALFF_hg38 is more conservative with a better precision and a lower number of false positives, preferring to maintain as much as fidelity to the original as possible (see, Table 2).

We can observe that although YALFF_hg38 has a smaller F-Measure compared to Quartz, the use of more informative k-mers dictionaries, e.g. YALFF_dbSNP and YALFF_All, increases the Fmeasure to comparable values. In particular, these two k-mers dictionaries appear to be only one that can improve the precision with respect to the unprocessed input. In summary, we can preserve an higher precision and a smaller number of false positives on SNP calling. This is very important in many medical applications where false positives should be avoided.

Table 2: Equivalent representation of Table 1 in terms of Precision, Recall and F-Measure.

\begin{tabular}{|c|c|c|c|}
\hline Method & Precision & Recall & F-Measure \\
\hline None & 0.9217 & 0.6341 & 0.7513 \\
Quartz & 0.9180 & 0.6520 & 0.7624 \\
YALFF_hg38 & 0.9216 & 0.6378 & 0.7539 \\
YALFF_Affy & 0.9216 & 0.6395 & 0.7551 \\
YALFF_dbSNP & 0.9223 & 0.6482 & 0.7613 \\
YALFF_All & 0.9219 & 0.6498 & 0.7623 \\
\hline
\end{tabular}

The reassembled dictionary of significant k-mers allows YALFF to inherit some characteristics of its rival. This can be easily noticed by looking at the increased recall. The larger is the k-mers database, the betters is the recall, reching its maximum with YALFF_All. Unfortunately, the precision does not follow a similar behavior, where YALFF_dbSNP is the best performing. The difference between the two smoothing algorithms account for the remaining differences, with Quartz being much more permissive in the definition of a modifiable quality value with YALFF being more conservative.

\subsection{Effects on Compression}

Another criteria of evaluation is the ability of the different tools to improve the compressibility of quality values. The effect on compression can be seen in last column of Table 3.

Table 3: The compression ratios are defined as $\frac{\text { uncompressed size }}{\text { compressed size }}$ obtained by compressing the resulting smoothed FASTQ using gzip, where the uncompressed size is $42 \mathrm{~GB}$. The size of the k-mer dictionary is also reported.

\begin{tabular}{|c|c|c|}
\hline Method & Index [GB] & Compression ratio \\
\hline None & 0 & 4.617 \\
Quartz & 19 & 6.925 \\
YALFF_hg38 & 5.26 & 7.147 \\
YALFF_Affy & 5.11 & 7.133 \\
YALFF_dbSNP & 6.27 & 7.241 \\
YALFF_All & 6.7 & 7.402 \\
\hline
\end{tabular}

First, we can observe that the processed FASTQ file is more compressible than the original file. This is expected as all methods tend to reduce the entropy of quality values. The compression ratio of YALFF is generally higher that Quartz, even if using just the hg38 genome as reference. Moreover, if more advanced k-mer dictionaries are used, then the compression ratio increases even further.

The most evident advantage of using a compressed index is the huge reduction of the dictionary size meaning less RAM used during execution. Quartz needs a powerful computer to run with large amount of memory, at least 32GB of RAM, whereas YALFF requires about 6-7GB of RAM, depending on the k-mers dictionary. It must be noted that it is possible to reassemble all the unique k-mers in the hg38 reference into a more compact form. This solution is not shown here because it is exactly equivalent in terms of performances to the standard hg38 dictionary which is more straightforward to compute, not involving the reassembly step. The only advantage is a reduced dimension of the k-mer dictionary from 5.26 GB to 5.09 GB.

The second advantage is the ability to store a large number of additional variations in linear space. For example, the total number of SNPs reported in the popular dbSNPs and Affymetrix SNPs are about 13M. We have shown that the hg38 and all these $13 \mathrm{M} \mathrm{mu-}$ tations can be indexed with as little as 6.7GB.

\subsection{Running Time}

While the reassembly of multiple k-mers into a single linear reference allows for reduced memory con- 
sumption and the possibility to use widespread indexing algorithms such as the FM-Index it also comes with reduced query speeds. The poor locality of succinct data structures, to which the FM-Index belongs, introduces a less efficient use of the cache. This may impact the execution time of YALFF making it about $1.3 x$ times slower than Quartz. This problem can be mitigated using some optimization tricks such as using multiple cores or extending a match on a kmer to search the neighbors on the linear sequence effectively reducing the number of accesses to the database.

\section{CONCLUSIONS AND FUTURE WORK}

This work has demonstrated the feasibility of combining a reassembly procedure with a string indexing algorithm to produce a very compact dictionary of kmers which works as drop-in replacements whenever static k-mer hash tables are needed. Given a SNPs database, like dbSNPs or Affymetrix SNPs, it is possible to find a set of $\mathrm{k}$-mers that are uniquely associated to a SNP. This set of k-mers can be efficiently compressed into a string dictionary and used for quality value compression. These $\mathrm{k}$-mers dictionaries are more informative than a single reference genome and they show better performance in terms of compression ratio and accuracy of genotyping, while keeping low memory requirements.

Future directions of research are the construction of a dynamic FM-Index with the ability to add and remove k-mers without recomputing the whole structure, another interesting problem is how to speed-up the search queries reducing cache misses. In the field of metagenomic read classification most methods are based on k-mers indexes (Girotto et al., 2017; Marchiori and Comin, 2017; Qian et al., 2018), and only recently the FM-index has been applied (Břinda et al., 2017), similarly the discovery of SNPs without mapping based on FM-index has been proposed only recently (Prezza et al., 2018). We believe that the use of FM-index will be beneficial in other alignment-free applications like pan-genomics.

\section{REFERENCES}

Benoit, G., Lemaitre, C., Lavenier, D., Drezen, E., Dayris, T., Uricaru, R., and Rizk, G. (2015). Reference-free compression of high throughput sequencing data with a probabilistic de Bruijn graph. BMC Bioinformatics, 16:288.
Břinda, K. (2016). Novel computational techniques for mapping and classifying Next-Generation Sequencing data. $\mathrm{PhD}$ thesis, Université Paris-Est.

Břinda, K., Salikhov, K., Pignotti, S., and Kucherov, G. (2017). Prophyle: a phylogeny-based metagenomic classifier using the burrows-wheeler transform. Poster at HiTSeq 2017.

Bonfield, J. K. and Mahoney, M. V. (2013). Compression of fastq and sam format sequencing data. Plos one.

Burrows, M. and Wheeler, D. J. (1994). A block-sorting lossless data compression algorithm. Technical report, Digital Equipment Corporation.

Chikhi, R., Limasset, A., and Medvedev, P. (2016). Compacting de bruijn graphs from sequencing data quickly and in low memory. Bioinformatics, 32(12):i201i208.

Cánovas, R., Moffat, A., and Turpin, A. (2014). Lossy compression of quality scores in genomic data. Bioinformatics, 30(15):2130-2136.

Comin, M., Leoni, A., and Schimd, M. (2014). Qcluster: Extending alignment-free measures with quality values for reads clustering. In Brown, D. and Morgenstern, B., editors, Algorithms in Bioinformatics, pages 1-13, Berlin, Heidelberg. Springer Berlin Heidelberg.

Comin, M., Leoni, A., and Schimd, M. (2015). Clustering of reads with alignment-free measures and quality values. Algorithms for Molecular Biology, 10(1):1-10.

Consortium, T. . G. P. (2012). An integrated map of genetic variation from 1,092 human genomes. Nature, 491(7422):56-65.

Ewing, B., Hillier, L., Wendl, M. C., and Green, P. (1998). Base-Calling of Automated Sequencer Traces UsingPhred. I. Accuracy Assessment. Genome Research, 8(3):175-185.

Ferragina, P. and Manzini, G. (2000). Opportunistic Data Structures with Applications. In Proceedings of the 41 st Annual Symposium on Foundations of Computer Science, FOCS '00, pages 390-, Washington, DC, USA. IEEE Computer Society.

Ferragina, P. and Manzini, G. (2005). Indexing Compressed Text. J. ACM, 52(4):552-581.

Girotto, S., Comin, M., and Pizzi, C. (2017). Higher recall in metagenomic sequence classification exploiting overlapping reads. BMC Genomics, 18(10):917.

Girotto, S., Comin, M., and Pizzi, C. (2018a). Efficient computation of spaced seed hashing with block indexing. BMC Bioinformatics, 19(15):441.

Girotto, S., Comin, M., and Pizzi, C. (2018b). Fsh: fast spaced seed hashing exploiting adjacent hashes. Algorithms for Molecular Biology, 13(1):8.

Greenfield, D. L., Stegle, O., and Rrustemi, A. (2016). GeneCodeq: quality score compression and improved genotyping using a Bayesian framework. Bioinformatics (Oxford, England), 32(20):3124-3132.

HG38 (2018). Human reference genome (hg38). http:// hgdownload.cse.ucsc.edu/goldenpath/hg38/bigZips/.

Illumina8bin (2011). Quality scores for next-generation sequencing, illumina inc. Technical report, Illumina Inc. 
Li, H. and Durbin, R. (2009). Fast and accurate short read alignment with Burrows-Wheeler transform. Bioinformatics (Oxford, England), 25(14):1754-1760.

Li, H. and Durbin, R. (2010). Fast and accurate long-read alignment with Burrows-Wheeler transform. Bioinformatics (Oxford, England), 26(5):589-595.

Malysa, G., Hernaez, M., Ochoa, I., Rao, M., Ganesan, K., and Weissman, T. (2015). QVZ: lossy compression of quality values. Bioinformatics (Oxford, England), 31(19):3122-3129.

Marçais, G. and Kingsford, C. (2011). A fast, lock-free approach for efficient parallel counting of occurrences of k-mers. Bioinformatics, 27(6):764-770.

Marchiori, D. and Comin, M. (2017). Skraken: Fast and sensitive classification of short metagenomic reads based on filtering uninformative k-mers. In Proceedings of the 10th International Joint Conference on Biomedical Engineering Systems and Technologies - Volume 3: BIOINFORMATICS, (BIOSTEC 2017), pages 59-67. INSTICC, SciTePress.

Mohamadi, H., Chu, J., Vandervalk, B. P., and Birol, I. (2016). nthash: recursive nucleotide hashing. Bioinformatics, 32(22):3492-3494.

Ochoa, I., Asnani, H., Bharadia, D., Chowdhury, M., Weissman, T., and Yona, G. (2013). QualComp: a new lossy compressor for quality scores based on rate distortion theory. BMC bioinformatics, 14:187.

Ochoa, I., Hernaez, M., Goldfeder, R., Weissman, T., and Ashley, E. (2017). Effect of lossy compression of quality scores on variant calling. Briefings in Bioinformatics, 18(2):183-194.

Prezza, N., Pisanti, N., Sciortino, M., and Rosone, G. (2018). Detecting Mutations by eBWT. In Parida, L. and Ukkonen, E., editors, 18th International Workshop on Algorithms in Bioinformatics (WABI 2018), volume 113 of Leibniz International Proceedings in Informatics (LIPIcs), pages 3:1-3:15, Dagstuhl, Germany. Schloss Dagstuhl-Leibniz-Zentrum fuer Informatik.

ProphAsm. Compressing k-mer sets via assembling contigs. https://github.com/prophyle/prophasm.

Qian, J., Marchiori, D., and Comin, M. (2018). Fast and sensitive classification of short metagenomic reads with skraken. Communications in Computer and Information Science, 881:212-226.

Rizk, G., Lavenier, D., and Chikhi, R. (2013). Dsk: k-mer counting with very low memory usage. Bioinformatics, 29(5):652-653.

Schimd, M. and Comin, M. (2016). Fast comparison of genomic and meta-genomic reads with alignment-free measures based on quality values. BMC Medical Genomics, 9(1):41-50.

Shajii, A., Yorukoglu, D., William Yu, Y., and Berger, B. (2016). Fast genotyping of known snps through approximate k-mer matching. Bioinformatics, 32(17):i538-i544.

Sherry, S. T., Ward, M.-H., Kholodov, M., Baker, J., Phan, L., Smigielski, E. M., and Sirotkin, K. (2001). dbsnp: the ncbi database of genetic variation. Nucleic Acids Research, 29(1):308-311.
Shibuya, Y. and Comin, M. (2018). Better quality score compression through sequence-based quality smoothing. 15th Annual Meeting of the Bioinformatics Italian Society. Submitted to BMC Bioinformatics.

SNPs (2018). List of known variants of the sample na12878. ftp://ussd-ftp.illumina.com/2017-1.0/hg38.

Yu, Y. W., Yorukoglu, D., Peng, J., and Berger, B. (2015). Quality score compression improves genotyping accuracy. Nature Biotechnology, 33(3):240-243.

Zook, J. M., Chapman, B., Wang, J., Mittelman, D., Hofmann, O., Hide, W. A., and Salit, M. L. (2014). Integrating human sequence data sets provides a resource of benchmark snp and indel genotype calls. Nature Biotechnology, 32:246-251. 